\title{
Distributed Energy-Efficient Clustering algorithm for Wireless Sensor Networks
}

\author{
K. Munusamy ${ }^{1}$, Dr. R. M. S. Paravathi ${ }^{2}$, M. Renuka ${ }^{3}$ \\ ${ }^{1}$ Research Scholoar, Department of Computer science and Engineering, Anna University, \\ Mahendra Institute of Engineering and Technology, Tiruchengode, TamilNadu Salem, India \\ ${ }^{2}$ Principal, Department of Computer science and Engineering Anna University, \\ Senguthar College of Engineering, Tiruchengode, TamilNadu Salem, \\ ${ }^{3}$ Asst.Professor, Department of Applied Science, Anna University, \\ S.S.M College of Engineering, Komarapalayam, TamilNadu Namakkal India
}

\begin{abstract}
Wireless sensor networks (WSNs) consist of a large number of limited capability (power and processing) Micro Electro Mechanical Systems (MEMS) capable of measuring and reporting physical variables related to their environment. In surveillance applications, sensors are deployed in a certain field to detect and report events like presence, movement, or intrusion in the monitored area. Minimizing energy dissipation and maximizing network lifetime are important issues in the design of applications and protocols for sensor networks. Energy-efficient sensor state planning consists in finding an optimal assignment of states to sensors in order to maximize network lifetime.

The existing scheme developed a centralized mechanism for near-optimal state assignment to sensors in large-scale cluster-based monitoring wireless sensor networks. The existing one was based on a tabu algorithm that computes a near-optimal network configuration in which each sensor can be activated, put in sleep mode or promoted as cluster head. The existing mechanism maximizes network lifetime while ensuring the full coverage of the monitored area and the connectivity of the obtained configuration. Connectivity is fulfilled through an optimally computed spanning tree connecting all the cluster heads. Due to abnormal node distribution in case of land surveillance, the existing tabu based optimal energy setting become complex. In addition the tabu algorithm keeps the probabilistic event detection independent for the respective node.

To overcome the abnormal node Distribution event detection triviality, distributed energy efficient algorithm is proposed in this work. The proposed work of this thesis, develop a more sophisticated heuristic to improve the network lifetime. The proposed scheme handles distance-dependent probabilistic event detection. The distance based probability is a function of the distance of the corresponding sensor from the event. The proposed system develop distributed algorithm which addresses the energy-efficient clustering under the joint coverage and routing constraint. The experimental simulations are carried for the proposed model using Network Simulator 2 (NS-2) for multiple simulation times, routing topology and energy coverage area.
\end{abstract}

\section{Introduction}

Wireless sensor networks (WSNs) consist of a large number of limited capability (power and processing) MicroElectroMechanical Systems (MEMS) capable of measuring and reporting physical variables related to their environment. In surveillance applications, sensors are deployed in a certain field to detect and report events like presence, movement, or intrusion in the monitored area. As depicted in Fig. 1, data collected by sensors are transmitted to a special node equipped with higher energy and processing capabilities called "processing node" (PN) or "sink". The PN collects, filters, and compiles data sent by sensors in order to extract useful information. Due to their energy constraints, wireless sensors usually have a limited transmission range, making multihop data routing toward the PN more energy efficient than direct transmission (one hop). Energy conservation in WSN is critical and has been addressed by substantial research. Generally, energy conservation is dealt with on five different levels

a. Efficient scheduling of sensor states to alternate between sleep and active modes

b. Energy-efficient routing, clustering, and data aggregation

c. Efficient control of transmission power to ensure an optimal trade-off between energy consumption and connectivity.

d. Data compression (source coding) to reduce the amount of uselessly transmitted data;

e. Efficient channel access and packet retransmission protocols on the Data Link Layer.

The scope of this work includes both the first and the second levels. We address the global problem of maximizing network lifetime under the joint clustering, routing, and coverage constraint. We consider a sensor 
network that is deployed in a certain area A to monitor some given events. When the network is dense, sensing ranges of neighbor sensors usually overlap.

This means that when an event occurs at a point $\mathrm{P}$ of $\mathrm{A}$, it will be detected and reported by all the sensors whose sensing range encompasses P. This redundant transmission results in useless energy consumption. To save network energy and increase its lifetime, we propose to switch on only a subset of sensors that covers A while all other sensors are turned off. On the other hand, clustering has been proven energy efficient in WSN. In cluster-based WSN, sensors are organized in clusters each having one sensor promoted as $\mathrm{CH}$. All non-CH nodes transmit their data to their $\mathrm{CH}$, which routes it to the remote PN. Clustering can provide for substantial energy saving since only $\mathrm{CH}$ sensors are involved in routing and relaying data. Moreover, clustering alleviates bandwidth, enables its reuse, and can, thus, increase system capacity. Besides, the fact that only the $\mathrm{CH}$ is transmitting information out of the cluster helps avoid collisions between the sensors inside the cluster and helps avoid the uncovered hole problem. However, since CHs consume more energy in aggregating and routing data, it is important to have an energy -efficient mechanism for CHs' election and rotation. In flat networks, sensors route data to the PN through their peer sensors using one of the many routing protocols.

In contrast, in clustered networks CHs transmit aggregated data to the PN, either directly (one hop) or in multi-hop. For the sake of minimizing energy consumption, both optimal number and optimal placement of CHs have to be sought. In a cluster-based monitoring sensor network, any energy-efficient sensor scheduling mechanism has to guarantee a certain area coverage rate. Besides, the connectivity of every sensor to a $\mathrm{CH}$ has to be ensured at any time.

Furthermore, for data to be routed from any $\mathrm{CH}$ to the $\mathrm{PN}$, all $\mathrm{CHs}$ have to belong to a single connected graph. Hence, for sensors' states allocation to be optimal, coverage, connectivity of sensors to $\mathrm{CHs}$, and routing have to be taken into account within the same global planning process. When coverage and connectivity are dealt with separately, the obtained configuration may not be optimal. For example, an optimal covering subset of sensors can fail to guarantee network connectivity because some nodes are switched off or the optimally designated CHs may belong to the set of switched-off sensors. Many works addressed separately energyefficient routing, clustering, and area coverage. Many other works addressed the integrated problem of maintaining area coverage and network connectivity but only on flat networks and did not take advantage of the potential energy saving and ease of manageability of cluster-based networks. To the best of our knowledge, the problem of maximizing sensor network lifetime under the integrated constraint of clustering, coverage, and routing has not been addressed within the same global optimization process.

\subsection{Existing System}

\section{Problem Definition}

The problem of maintaining both area coverage and network connectivity under energy constraint in WSN has been extensively addressed in the literature and many protocols were proposed to alternate sensor states between active and sleep in order to maximize network lifetime. For example, Xing et al. provides a geometric analysis of the relationship between coverage and connectivity, and propose the Coverage Configuration Protocol (CCP) that dynamically configures the network to guarantee different degrees of coverage depending on the application requirements. In CCP, every node decides its state (Active or Sleep) based on the coverage degree of the intersection points of its sensing circle with those of its neighbors. When coupled with any connectivity maintenance protocol, $\mathrm{CCP}$ offers connectivity and $\mathrm{K}$-coverage. Lu et al. presents Scalable Coverage Maintenance (SCOM), a localized coverage maintenance algorithm where sensors use the same redundancy eligibility rule as in to decide whether to turn on or turn off. SCOM implements, for each sensor, a back-off timer proportional to its residual energy. The back-off timer allows sensors with lower residual energies to decide about their states before sensors with more energy, making them more likely to turn off than the other sensors, if they find themselves redundant. Chamam and Pierre propose a centralized heuristic which dynamically calculates a near-optimal subset of sensors that guarantees a predefined coverage rate while ensuring network connectivity when the transmission range is greater than or equal to twice the sensing range. Yan et al. propose to schedule sensors' activities (Active/Sleep) so that every point in a grid-monitored area is covered at any time. Neighbor sensors exchange a random reference time Tref within cyclic rounds of constant duration $\mathrm{T}$ and decide to be active for a certain time duration within $\mathrm{T}$. The round period $\mathrm{T}$ is equally shared among all the neighbor sensors that cover a common grid. Even though the proposed schedule balances consumed energy over neighbor nodes, it does not take into account the residual energy of sensors when calculating the activation time period of every node, which makes nodes with less residual energy more prone to expiration. However, all the works cited above do not address cluster-based architectures. Cluster formation is typically based on the energy reserve of sensors and sensors proximity to the cluster head. Energy-efficient cluster-based routing algorithms for WSN have been widely addressed in literature.. For instance, Low-Energy Adaptive Clustering Hierarchy (LEACH), one of the most popular hierarchical routing algorithms for WSN, proposes to form clusters of sensor nodes based on the received signal strength and use local cluster heads as routers to the sink. This saves energy since the transmissions will only be operated by cluster heads rather than 
all sensor nodes. Even though LEACH is completely distributed, it uses single-hop communication between cluster heads and the sink, which is energy consuming and not applicable to networks deployed in large regions. Power-Efficient Gathering in Sensor Information Systems (PEGASIS) and its variant Hierarchical-PEGASIS are two improvements of LEACH. Rather than forming multiple clusters, PEGASIS forms chains of sensor nodes so that each node transmits to and receives from a neighbor and only one node is selected from that chain to transmit to the PN. But still, communication between the elected CH and the PN is made in one hop, which is not suitable for large networks.

\subsection{Proposed System}

In this work, we address the optimal planning of cluster-based WSN under the joint routing and coverage constraint. In our architecture, any sensor can be active, switched off, or upraised as $\mathrm{CH}$, and only CHs can route data. We seek an optimal allocation of states to sensors, which maximizes network lifetime, while ensuring simultaneously full area coverage, connectivity of every sensor to a $\mathrm{CH}$, and connectivity of the overlay network composed of CHs.

Minimizing energy dissipation and maximizing network lifetime are important issues in the design of applications and protocols for sensor networks. Energy-efficient sensor state planning consists in finding an optimal assignment of states to sensors in order to maximize network lifetime. For example, in area surveillance applications, only an optimal subset of sensors that fully covers the monitored area can be switched on while the other sensors are turned off. In this work, we address the optimal planning of sensors' states in cluster-based sensor networks. Typically, any sensor can be turned on, turned off, or promoted cluster head, and a different power consumption level is associated with each of these states. We seek an energy-optimal topology that maximizes network lifetime while ensuring simultaneously full area coverage and sensor connectivity to cluster heads, which are constrained to form a spanning tree used as a routing topology. First, we formulate this problem as an Integer Linear Programming model that we prove NP-Complete. Then, we implement a Tabu search heuristic to tackle the exponentially increasing computation time of the exact resolution.

Experimental results show that the proposed heuristic provides near-optimal network lifetime values within low computation times, which is, in practice, suitable for large-sized sensor networks.

\section{Proposed Heuristic}

Our problem consists in finding the optimal allocation of states to sensors, which maximizes network lifetime under the integrated constraint of coverage, clustering, and routing. We call this problem OPT-ALLRCC. To maximize network lifetime, we need a trade-off between total energy consumption and energy balancing among sensors. For example, to ensure area coverage, we would prefer to activate more sensors having higher residual energy (and consuming a higher total energy) than few sensors having little residual energy (consuming less total energy but more prone to expiration). O On the other hand, any admissible solution of our model has to ensure full coverage of the monitored area and the existence of a spanning tree connecting all CHs. To model this problem, we first define the following sets and constants.

As the considered problem is NP-Complete, we propose a Tabu search heuristic, called TABU-RCC, to tackle to exponentially increasing processing time of the exact solution. TABU-RCC will be run by the PN to find a near-optimal sensor state configuration. As shown in Algorithm 1, TABU-RCC starts with an admissible solution and iteratively performs movements that consist in changing the state of one sensor at a time. The best solution found after the predefined number of iterations is transposed on sensors to form the new network configuration. The network will operate with this configuration for a predefined period T during which residual energies of active nodes and CHs will decrease, then TABU-RCC is run again to find a new configuration based on the new values of residual energies. This new configuration will be kept for another period $\mathrm{T}$ and so forth. The periodic execution of TABU-RCC by the PN requires sensors-related information (e.g., residual energies) to be transmitted periodically to the PN (upstream communication) and the newly computed sensor states to be transmitted to the sensors (downstream communication). In our architecture, sensor-related data will be collected exactly in the same manner as the sensed data, i.e., using the cluster-based hierarchical structure of the network. Active sensors that have data to report will send it to the PN via their respective CHs. They will append the value of their respective residual energies to the data packets they are sending. When they have no data to send, they will synchronize their energy information with the PN.

\subsection{Wireless Sensor Networks}

\section{Tabu Search in Wireless Sensor Networks}

The wireless sensor network (WSN) consists of spatially distributed autonomous sensors to cooperatively monitor physical or environmental conditions, such as temperature, sound vibration, pressure, motion or pollutants. The development of wireless sensor networks was motivated by military applications such as battlefield surveillance. They are now used in many industrial and civilian application areas, including 
industrial process monitoring and control, machine health monitoring, environment and habitat monitoring, healthcare applications, home automation, and traffic control.

In addition to one or more sensors, each node in a sensor network is typically equipped with a radio transceiver or other wireless communications device, a small microcontroller, and an energy source, usually a battery. A sensor node might vary in size from that of a shoebox down to the size of a grain of dust, although functioning "motes" of genuine microscopic dimensions have yet to be created. The cost of sensor nodes is similarly variable, ranging from hundreds of dollars to a few pennies, depending on the size of the sensor network and the complexity required of individual sensor nodes. Size and cost constraints on sensor nodes result in corresponding constraints on resources such as energy, memory, computational speed and bandwidth. A sensor network normally constitutes a wireless ad-hoc network, meaning that each sensor supports a multi-hop routing

Algorithm (several nodes may forward data packets to the base station).Our approach makes use of the computational capabilities of each sensor node to predict the possible locations of the target over time. The measurements and predictions from each of the sensors are combined at a single designated node. For example, given a known maximum detection radius and the time when a sensor first encounters the tracked object, it is safe to assume that the tracked object is at the maximum detection distance from the sensor. If the tracked object moves according to a linear trajectory, the longer the duration that the object spends in a sensor's detection region, the closer it would have had to pass by that sensor. We build on this idea to estimate the distance from a sensor to the object. Thus we calculate its approximate location with a weighted average of the detecting sensors' coordinates, where the inverses of these distance estimates are used as weights. This weighted average can be computed efficiently inside the network during data aggregation. In order to predict the movement of the subject over time, we fit a linear trajectory estimate to the set of most recent location estimates

\subsection{Energy Efficient Cluster}

There have been several network routing protocols proposed for wireless networks that can be examined in the context of wireless sensor networks. We examine two such protocols, namely direct communication with the base station and minimum-energy multi-hop routing using our sensor network and radio models. In addition, we discuss a

conventional clustering approach to routing and the drawbacks of using such an approach when the nodes are all energy-constrained.Using a direct communication protocol, each sensor sends its data directly to the base station. If the base station is far away from the nodes, direct communication will require a large amount of transmit power from each node. This will quickly drain the battery of the nodes and reduce the system lifetime. However, the only receptions in this protocol occur at the base station, so if either the base station is close to the nodes, or the energy required to receive data is large, this may be an acceptable (and possibly optimal) method of communication.

The second conventional approach we consider is a "minimum-energy" routing protocol. There are several power-aware routing protocols. In these protocols, nodes route data destined ultimately for the base station through intermediate nodes. Thus nodes act as routers for other nodes' data in addition to sensing the environment. These protocols differ in the way the routes are chosen. Some of these protocols only consider the energy of the transmitter and neglect the energy dissipation of the receivers in determining the routes. In this case, the intermediate nodes are chosen such that the transmit amplifier energy is minimized thus node However, for this minimum-transmission-energy (MTE) routing protocol, rather than just one (high-energy) transmit of the data, each data message must go through $\mathrm{n}$ (lowenergy) transmits and $\mathrm{n}$ receives. Depending on the relative costs of the transmit amplifier and the radio electronics, the total energy expended in the system might actually be greater using MTE routing than direct transmission to the base station. In MTE routing, each node sends a message to the closest node on the way to the base station. Thus the node located a distance nr from the base station would require $n$ transmits a distance $r$ and $n-1$ receives.

\subsubsection{Construction of clusters}

The construction of clusters follows the idea of a greedy algorithm, where nodes choose a neighbor with higher capability grade as parent, while other nodes that do not have such a neighbor are roots. The message SetRoot is used for propagating the address of the root node to all the members of the clusters. The Initialization phase and the event SetRoot from Algorithm 1 give a formal description for the construction of clusters. Briefly, the protocol works as follows:

Nodes that have the highest capability grades among their neighbors declare themselves clusterheads and broadcast a SetRoot message announcing their roles.The remaining nodes choose as parent the neighbor with the highest capability grade. When a node receives a SetRoot message from its parent, it learns the cluster membership and rebroadcasts the SetRoot message. 


\subsubsection{Knowledge on adjacent clusters}

Once the clustering structure is set up, the root nodes need to establish links to the adjacent clusters. The root nodes learn about the adjacent clusters from the nodes placed at the cluster borders. During the propagation of the broadcast message SetRoot down to the leaf nodes, the message is also received by nodes from adjacent clusters. These nodes store the adjacent root identity in their $\mathrm{Ru}(\mathrm{v})$ sets and report it to their parents. The information is propagated up in the tree with a message which we term UpdateInfo. Through this message, nodes learn the next hops for the paths leading to the clusters adjacent to their sub-trees. In particular, the root nodes learn the adjacent clusters and the next hops on the paths to reach their clusterheads. The events of receiving messages SetRoot and UpdateInfo from Algorithm 1 describe how the knowledge and the paths to adjacent clusters is updated for a given node v. Duplicate UpdateInfo messages are discarded: a node $v$ sends the message UpdateInfo to its parent if and only if the set of known root nodes changes. This means that if $\mathrm{v}$ is informed about a root node from one neighbor, but it knows already about this root through another neighbor, $\mathrm{v}$ does not propagate the information to the parent again.

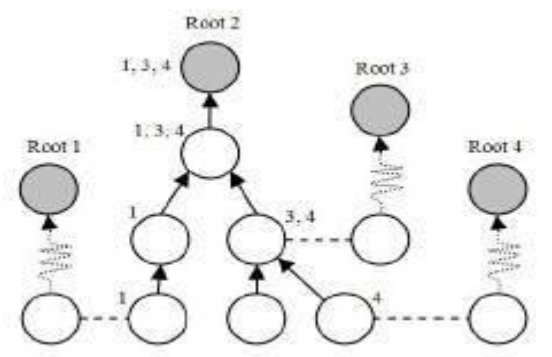

Fig 1: Nodes learn from neighbors which are the adjacent clusters and propagate the knowledge to the parents.

\subsubsection{Maintenance in face of topology changes}

We analyze how the clustering structure adapts to dynamic environments. We term the events regarding topology changes Link Add and Link Delete. Algorithm 1 gives a detailed description of the behavior of node $\mathrm{v}$ when these events occur. In short, there are two situations where nodes adjust their cluster membership:

A node discovers a new neighbor with a higher capability grade than its current parent. The node then selects that neighbor as its new parent.A node detects the failure of the link to its parent. The node then chooses as new parent the node with the highest capability grade in its neighborhood. Besides reclustering, topology changes may also require modifications in the knowledge on adjacent clusters. The SetRoot message informs nodes about the cluster membership of their neighbors, while the UpdateInfo message is used for transmitting the updates from children to their parents. We distinguish the following situations:

A node $\mathrm{v}$ detects a new neighbor from a different cluster. Consequently, $\mathrm{v}$ adds the root of that cluster to its knowledge.A node $\mathrm{v}$ switches from parent $\mathrm{p} 0$ to $\mathrm{p} 1$. Then $\mathrm{v}(1)$ notifies $\mathrm{p} 0$ to remove the information associated with $\mathrm{v}$ and (2) sends the list of adjacent clusters to p1.A node $\mathrm{v}$ detects the failure of the link to one of its neighbors $u$. As a result, $v$ erases the knowledge associated with $u$.Any change of global knowledge at node $v$ results in transmitting the message UpdateInfo from $\mathrm{v}$ to its parent.

Tabu search is a metaheuristic algorithm that can be used for solving combinatorial optimization problems, such as the traveling salesman proble (TSP). Tabu search uses a local or neighbourhood search procedure to iteratively move from a solution $x$ to a solution $x^{\prime}$ in the neighbourhood of $x$, until some stopping criterion has been satisfied. To explore regions of the search space that would be left unexplored by the local search procedure (see local optimality), tabu search modifies the neighbourhood structure of each solution as the search progresses. The solutions admitted to $N^{*}(x)$, the new neighbourhood, are determined through the use of memory structures. The search then progresses by iteratively moving from a solution $x$ to a solution $x^{\prime}$ in $N^{*}(x)$.

Perhaps the most important type of memory structure used to determine the solutions admitted to $N^{*}$ $(x)$ is the tabu list. In its simplest form, a tabu list is a short-term memory which contains the solutions that have been visited in the recent past (less than $n$ iterations ago, where $n$ is the number of previous solutions to be stored ( $n$ is also called the tabu tenure)). Tabu search excludes solutions in the tabu list from $N^{*}(x)$. A variation of a tabu list prohibits solutions that have certain attributes or prevent certain moves (e.g. an arc that was added to a TSP tour cannot be removed in the next $n$ moves). Selected attributes in solutions recently visited are alabeled "tabu-active." Solutions that

contain tabu-active elements are "tabu". This type of short-term memory is also called "recency-based" memory.Tabu lists containing attributes can be more effective for some domains, although they raise a new problem. When a single attribute is marked as tabu, this typically results in more than one solution being tabu. Some of these solutions that must now be avoided could be of excellent quality and might not have been visited. 
To mitigate this problem, "aspiration criteria" are introduced: these override a solution's tabu state, thereby including the otherwise-excluded solution in the allowed set. A commonly used aspiration criterion is to allow solutions which are better than the currently-known best solution

\section{Performance Evaluation}

To evaluate our TABU-RCC in terms of network lifetime, we compared it to EESH. EESH functions as follows: nodes are promoted cluster heads according to their respective residual energies, their respective degrees, the distance to their neighbors, and the residual energies of these neighbors. For that, EESH evaluates a cost function for every sensor in the network and iteratively elects the node having the greatest cost as $\mathrm{CH}$. This process terminates when all non-CH sensors in the network are connected to at least one cluster head. As EESH does not consider optimal area coverage, we made a small modification of EESH that consists of computing, in each cluster provided by EESH, an optimal subset of non-CH sensors that will be activated while the other sensors are turned off. Fig. 10 depicts the network lifetime provided, respectively, by TABU-RCC, EESH, and the modified version of EESH, for different network sizes. We clearly see that TABU-RCC outperforms the modified version of EESH by providing three times longer network lifetime, for all network sizes. This considerable gain is one of the main benefits of our centralized heuristic.

\section{Conclusion}

The proposed model provided a centralized mechanism for near-optimal state assignment to sensors in large-scale cluster-based monitoring wireless sensor networks. Our mechanism is based on a tabu algorithm that computes a near-optimal network configuration in which each sensor can be activated, put in sleep mode or promoted as cluster head. Our mechanism maximizes network lifetime while ensuring the full coverage of the monitored area and the connectivity of the obtained configuration. Connectivity is fulfilled through an optimally computed spanning tree connecting all the cluster heads. Simulations show that our mechanism provides for acceptable results with respect to the exact solutions of the derived ILP model, within low computation times. Despite its centralized aspect, our mechanism exhibits low complexity and low computation times making its practical implementation adaptable for large-scale networks.

As future research directions, we intend to develop a more sophisticated heuristic to improve the network lifetime. Furthermore, we intend to consider distance-dependent probabilistic event detection, where the probability that a sensor detects an event is function of the distance of that sensor from the event. Furthermore, we intend to work on distributed algorithms that address energy-efficient clustering under the joint coverage and routing constraint.

\section{References}

[1] C.Y. Chong and S.P. Kumar, "Sensor Networks: Evolution, Opportunities, and Challenges," Proc. IEEE, vol. 91, no. 8, pp. $1247-$ 1256, Aug. 2003

[2] M. Cardei, M.T. Thai, Y. Li, and W. Wu, "Energy-Efficient Target Coverage in Wireless Sensor Networks," Proc. IEEE INFOCOM, vol. 3, pp. 1976-1984, 2005.

[3] V. Raghunathan, C. Schurgers, S. Park, and M.B. Srivastava, "Energy-Aware Wireless Microsensor Networks," IEEE Signal Processing Magazine, vol. 19, pp. 40-50, 2002.

[4] K. Akkaya and M. Younis, "A Survey of Routing Protocols in Wireless Sensor Networks," Ad Hoc Network J., vol. 3, no. 3, pp. 325-349, 2005.

[5] L.M.C. Arboleda and N. Nasser, "Comparison of Clustering Algorithms and Protocols for Wireless Sensor Networks," Proc. Canadian Conf. Electrical and Computer Eng., pp. 1787-1792, May 2006.

[6] M. Younis, P. Munshi, G. Gupta, and S.M. Elsharkawy, "On Efficient Clustering of Wireless Sensor Networks," Proc. Second IEEE Workshop Dependability and Security in Sensor Networks and Systems, pp. 78-91, 2006.

[7] H. Karl and A. Wiling, Protocols and Architectures for Wireless Sensor Networks. Wiley, 2005.

[8] Y. Yao and G.B. Giannakis, "Energy-Efficient Scheduling for Wireless Sensor Networks," IEEE Trans. Comm., vol. 53, no. 8, pp. $1333-1342,2005$.

[9] S.F. Hwang, Y.Y. Su, Y.Y. Lin, and C.R. Dow, "A Cluster-Based Coverage-Preserved Node Scheduling Scheme in Wireless Sensor Networks," Proc. Third Ann. Int'l Conf. Mobile and Ubiquitous Systems: Networking and Services, pp. 1-7, July 2006.

[10] W.R. Heizelman, A. Chandrakasan, and H. Balakrishnan, "Energy-Efficient Communication Protocol for Wireless Micro Sensor Networks," Proc. IEEE Hawaii Int'l Conf. System Sciences, Jan. 2000.

[11] V. Mhatre and C. Rosenberg, "Homogeneous vs. Heterogeneous Clustered Sensor Networks: A Comparative Study," Proc. IEEE Int'l Conf. Comm., vol. 6, pp. 3646-3651, June 2004.

[12] M. Inanc, M.I. Magdon, and B. Yener, "Power Optimal Connectivity and Coverage in Wireless Sensor Networks," Technical Report TR-03-06, Dept. of Computer Science, Rensselaer Polytechnic Inst., 2003.

[13] J. Carle and D. Simplot, "Energy Efficient Area Monitoring by Sensor Networks," Computer, vol. 37, no. 2, pp. 40-46, Feb. 2004.

[14] X.Y. Li, P.J. Wan, and O. Frieder, "Coverage in Wireless Ad-Hoc Sensor Networks," IEEE Trans. Computers, vol. 52, no. 6, pp. 753- 763, June 2002.

[15] G. Xing, X. Wang, Y. Zhang, C. Lu, R. Pless, and C. Gill, "Integrated Coverage and Connectivity Configuration for Energy Conservation in Sensor Networks,” ACM Trans. Sensor Networks, vol. 1, no. 1, pp. 36-72, Aug. 2005. 


\section{Authors}

K.Munusamy received: his M.C.A. Degree in Computer Applications, from K.S.R College of Technology 2004 at Periyar University, Salem and M.Phil degree in Computer Science -2008, Salem at Periyar University and M.E. degree in Computer science and Engineering from Jaya College of Engineering and Technology, ANNA University, Coimbatore .Currently He is doing his research in the field of Wireless Sensor Networks in Bharathiyar University, Coimbatore, India. He fields of interests are Wireless Networks; Wireless He has published 1 paper in International conferences

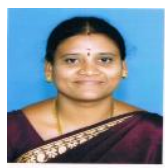

M.Renuka: received the Bachelor of Science Degree in Computer Science from Madras University in 1999 and her Master Degree in Computer Application from the Bharathidasan University in 2002. She completed her M.Phil Degree in the year 2005 from Periyar University. She is pursuing Ph.D.,in Computer Science at Mother Teresa University. Currently working as a Assistant Professor in the Department of Applied Science at SSM College of Engineering, Komarapalayam. She is presently working in the area of Mobile Security. Other areas of interest include Design and Analysis of Algorithms, Software Engineering and Extreme Programming. 